\title{
RECENT DEVELOPMENT IN THE THEORY OF LINEAR PARTIAL DIFFERENTIAL EQUATIONS
}

\author{
JEAN DIEUDONNÉ \\ Villa Orangini \\ 119 Avenue de Braucolar \\ Nice, France 06100 \\ (Received French Manuscript on July 1i, 1978) \\ Translated by J. N. PANDEY \\ Mathematics Department \\ Carlton University \\ Ottawa, Canada
}

ABSTRACT. A historical development of the theory of linear partial differential equation is reviewed with comments. A recent development in the theory of linear partial differential equations is discussed.

KEY WORDS AND PHRASES. Linear Partial Differential Equations, Spectral Theory, Theory of Distributions, Pseudo-Differential Operator, Elliptic Differential Operator, Cauchy Problem.

1980 MATHEMATICS SUBJECT CLASSIFICATION CODES. 35-02, 35A05, 35 S05.

1. INTRODUCTION. 
The history of linear partial differential equations is more than two centuries old; it is closely related with the development of functional analysis, in which it is a source of very important notions such as spectral theory and the theory of distributions. It is remarkable that this history is full of a series of pitfalls.

2. It was natural to think that ordinary differential equations would give a model of the behavior of solutions which would later generalize to partial differential equations. The paradigm in this connection was the local uniqueness and existence theorem of Cauchy for a vector-valued differential equation

$$
y^{\prime}=A(x, y)
$$

where $x$ is a real variable, $y$ a vector in $R^{n}$, $A$ is a mapping from $I x H$ to $H$, $I$ is an interval of $R$ and $H$ is an open subset of $R^{n}$. In fact a general theorem of such a nature is the theorem of Cauchy-Kowalewski and Hölmgren: Let us consider the system of $r$ equations in $r$ real-valued unknown functions $v_{1}$, $v_{2}, \ldots v_{r}$ of $p+1$ real variables $x_{1}, x_{2}, \ldots x_{p+1}$, of the form

$$
\frac{\partial v_{j}}{\partial v_{p+1}}=H_{j}\left(x_{1}, x_{2}, \ldots x_{p+1}, v_{1}, v_{2}, \ldots v_{r}, \frac{\partial v_{1}}{\partial x_{1}}, \frac{\partial v_{1}}{\partial x_{2}}, \ldots \frac{\partial v_{r}}{\partial x_{p-1}}, \frac{\partial v_{r}}{\partial x_{p}}\right),(1 \leq j \leq r)
$$

where the right-hand side expressions do not contain derivatives with respect to $\mathrm{x}_{\mathrm{p}+1}$, and $\mathrm{H}_{\mathrm{j}}$ are supposed to be real and analytic functions of $\mathrm{p}+1+\mathrm{r}+\mathrm{rp}$ variables in a neighbourhood $v_{0}$ of the origin in $\underline{R}^{p+1}+r+r p$. Under these conditions there exists a neighbourhood $\mathrm{V} \subset \mathrm{V}_{0}$ of the origin in $\mathrm{R}^{\mathrm{p}+1}$ and a solution $\left(v_{1}, \ldots v_{r}\right)$ of this system consisting of functions analytic in $v$ such that $v_{j}\left(x_{1}, \ldots . x_{p}, 0\right)=0$ for $1 \leq j \leq r$ in $V \cap \underline{R} \underline{p}$. Moreover if all $H_{j}$ are 
linear in all $v_{j}$ and $\frac{\partial v_{j}}{\partial x_{k}}$, this system of functions is the only system of $C^{\infty}$ class having such properties. For equations where the hypothesis of analyticity does not hold we have only one analogous theorem: the one concerning one single equation ( 1$)(\mathrm{r}=1)$ where the right hand $\mathrm{H}$ is real-valued and belongs to class $C^{\infty}$ with respect to the variables $x_{1}, x_{2}, \ldots x_{p+1}, V, \frac{\partial V}{\partial x_{1}}, \ldots \frac{\partial V}{\partial x_{p}}$. It was not till 1955 that it was shown that these results could not be generalized, thanks to a series of counter-examples: the first example being the celebrated example of $\mathrm{H}$. Lewy of a linear equation of order 1 with complex coefficients $\epsilon C^{\infty}$ (which is equivalent to a system of two real equations) having no solutions (even if distributional solutions are admitted); examples of equations such as

$$
\frac{\partial V}{\partial y}=a(x, y) \frac{\partial V}{\partial x} \text { where } a \text { is a complex valued } c^{\infty} \text {, function for }
$$

which the Cauchy problem with $\mathrm{V}(\mathrm{x}, 0)=0$ has more than one solution; finally the example by Plis of an elliptic equation in a ball, with $C^{\infty}$ - coefficients which does not have a global solution but has local solutions in the neighbourbood of every point.

3. From the work of Laplace, Fourier, Poisson and Cauchy attention was drawn towards linear equations with constant coefficients not only because they were important for application in Physics, but because the Fourier-Laplace transform reduces such problems to algebraic ones. If $\underline{\mathrm{p}} \cdot \mathrm{u} .=\sum \mathrm{A}_{\alpha} \mathrm{D}^{\alpha} \mathrm{u}$ where the $A_{\alpha}$ are constants, we have $\quad|\alpha| \leq m$

$$
F(P . u)(\xi)=\underset{|\alpha| \leq m}{\left(\sum_{\alpha}(2 \pi i \xi)^{\alpha}\right) \quad F u(\xi),}
$$

and from the equation P.u $=f$, we have 


$$
\begin{aligned}
& \left(\sum_{\alpha}^{A_{\alpha}}(2 \pi i \xi)^{\alpha}\right) \quad \underline{F} u(\xi)=\underline{F} f(\xi), \\
& |\alpha| \leq m
\end{aligned}
$$

provided the transforms of $\mathrm{u}$ and $\mathrm{f}$ exist; if $\mathrm{v}$ is a function satisfying this equation and if its inverse Fourier transform $\underline{\bar{F}} v$ exists it will be a solution to the equation $\underline{P} \cdot u=f$. The extension of the Fourier transformation to tempered distributions has further enlarged the scope of applicability of this method, permitting among other things the proof in all cases of the existence of elementary solutions satisfying $\mathrm{Pu}=\delta_{0}$ where $\delta_{0}$ is Dirac measure at the origin. Finally, a device introduced by Korn, which has been extensively used for the local study of linear equations with variable coefficients, consists in "approaching" the equation considered by replacing the coefficients by their values at the point $x_{0}$ in the neighbourhood of interest; from the knowledge of the solutions considered one then succeeds in many cases in deriving a solution of the original equation. But it is clear that most of the problems which are posed in the theory of linear partial differential equations are invariant under diffeomorphism (for example the Dirichlet problem with smooth boundary); it is therefore strange to emphasize a priori a class of equations which do not possess such an invariance property. Their consideration is justified for the problems which are only translation invariant (for example the Dirichlet problem for spheres); these then subordinate to the problem of differential operators on the homogeneous spaces $\mathrm{G} / \mathrm{H}$, which are invariant under $\mathrm{G}$.

4. Before 1940, the works on linear partial differential equations were mainly devoted to 2 nd order equations. For hyperbolic equations the generalization to higher order equations proceeds in a natural way, but for elliptic equations the existence (peculiar to 2nd order) of the maximum principle which permits an elaborate study of solutions, completely distorts the 
perspective when one wishes to pass to elliptic equations of arbitrary order. 5. From 1950 on, the theory of distributions, in conjunction with the spectral theory of Hilbert-Carleman-von Neumann, has permitted considerable progress, notably in the treatment of the boundary value problems for elliptic or hyperbolic equations of any order.

The main idea is to use the theory of distributions to construct function spaces (usually Hilbertian), the most important of which are the Sobolev spaces ${ }_{\mathrm{H}}^{\mathrm{p}}(\Omega)$; for an integer $\mathrm{p} \geq 0$, this space consists of distributions $\mathrm{T}$ on an open set $\Omega$ of $R^{n}$ such that $T$ and all its distributional derivatives $D^{\alpha} T$ for $|\alpha| \leq \mathrm{p}$ belong to $\mathrm{L}^{2}(\Omega)$; defining the square of the norm of $\mathrm{T}$ as the value $\sum_{|\alpha| \leq p} \int_{\Omega}\left|D^{\alpha} T\right|^{2} d x$ we obtain a Hilbert space. Let $P$ be a differential operator which to every $u \in C^{\infty}$ assigns the function $\sum_{\alpha} A_{\alpha}(x) D^{\alpha} U(x)$ where $A_{\alpha}$ $|\alpha| \leq m$

belongs to $\mathrm{C}^{\infty}$ in $\Omega$; then we define $\underline{\mathrm{P}}$. T for all distributions on $\Omega$ by the same formula, and then one may consider distributions $T$ satisfying $\underline{P} \cdot T=f$ for $a$ given function or distribution $\mathrm{f}$. Under certain restrictions on the operator $\underline{P}$ and on the given boundary values one can establish a priori inequalities of the type $\|\mathrm{u}\| \mathrm{E}_{1} \leq\|\mathrm{P} \cdot \mathrm{u}\|_{\mathrm{E}_{2}}+\|\mathrm{u}\|_{\mathrm{E}_{3}}$ for norms on suitable functional spaces $E_{j}$. By general results of functional analysis (based upon the Hahn-Banach theorem and general properties of Hilbert spaces), one can in many cases prove the existence and the uniqueness of solutions which are known a priori only to belong to the functional spaces under consideration. This method has become very popular among the specialists, who have often been satisfied with these "weak" solutions and given only secondary importance to proving that when $f \in C^{\infty}$, the weak solutions of $\underline{P} \cdot u=f$ should be genuine $C^{\infty}$ solutions satisfying in the classical sense the given boundary conditions. The proofs consist 
in difficult manipulations of a priori inequalities, using for instance, the fact that the intersection of the spaces $H^{m}(\Omega)$, as $m$ tends to $\infty$, is the space of $\mathrm{C}^{\infty}$ functions. It may also be noted that these methods are non-constructive even when they establish the uniqueness of the solution.

Nevertheless the results obtained by this method have been spectacular, so much so that by 1960 , the specialists had the impression that they had arrived at an almost final state of the general theory.

6. Now the perspective has completely changed; the books written on the subject during the period 1960-65 are outdated to a great extent. This is due to the introduction of a new technique: that of pseudo-differential operators and its generalizations.

One can associate the initial idea of this theory to the earlier results on the solution of certain boundary value problems for linear partial differential equations such as the Dirichlet problem for the Laplace equation or the Cauchy problem for wave equations; when such a problem $\underline{P} \cdot u=f$ has a unique solution we can express it in the form of an integral operator $\mathbf{u}=\underline{\mathrm{K}} \cdot \mathbf{f}$, where $(\underline{K} \cdot f)(x)=\int K(x, y) f(y) d y$ $\mathrm{K}$ being any integrable kernel function. It seems therefore that there is a fundamental difference in nature between a differential operator $\underline{P}$ and its inverse $\underline{K}$. Is it not possible to consider the differential and integral operators as if they all belong to a larger class of operators forming an algebra in which the notion of inverse would be the natural algebraic notion? This is an old idea which can be credited to Leibniz and which began to be realized in Riemann-Liouville's derivative of fractional order. The first reasonably general and modern attempt in this direction has been the theory 
of singular integral operators of Calderon-Zygmund, which introduced a significant link between the above theory and harmonic analysis. Towards 1963 this theory was considerably generalized by Kohn, Nirenberg, and Hormander; as for the derivative of Riemann-Liouville we can present its fundamental idea by writing a differential operator as an integral operator by means of the Fourier transformation. If $\underline{P}$ is the differential operator defined above where the $A_{\alpha}$ are $C^{\infty}$ functions in $R^{n}$, and if $\left.f \in \underline{S} \underline{(R}^{n}\right)$ we can write and it follows that

$$
D^{\alpha} f(x)=\int_{\underline{R}^{n}} e^{2 \pi i(x \mid \xi)}(2 \pi i \xi)^{\alpha} F f(\xi) d \xi
$$

$$
(\underline{p} \cdot f)(x)=\int_{\underline{R}^{n}} e^{2 \pi i(x \mid \xi)} \sigma_{\underline{P}}(x, \xi) F f(\xi) d \xi,
$$

where the symbol

$$
\sigma_{\underline{p}}(x, \xi)=\sum_{|\alpha| \leq m} A_{\alpha}(x)(2 \pi i \xi)^{\alpha},,
$$

is a polynomial of degree $m$ in $\xi \in R_{j}^{n}$ which we write $\sigma_{\underline{p}}=\sigma_{\underline{p}}^{o}+\sigma_{\underline{p}}^{1}$ where $\sigma_{\underline{p}}^{o}$ is homogeneous of degree $m$ in $\xi$ and $\sigma_{\underline{P}}^{1}$ is the sum of the terms of degree $<m$ in $\xi ; \sigma_{\underline{p}}^{\circ}$ is called "the principal part" of $\underline{P}$ and has the important property that if $Q$ is another differential operator we have.

$$
\sigma_{\underline{Q P}}^{o}=\sigma_{\underline{Q}}^{o} \cdot \sigma_{\underline{P}}^{o}
$$

The generalization consists in replacing the symbol in (2) by a more general $C^{\infty}$ function with prescribed growth condition as $|\xi|$ tends to infinity: for the symbol $\sigma_{\underline{p}}$ of the form (3), $D_{x}^{\alpha} \sigma_{\underline{p}}$ has the same asymptotic order in $\xi$ as $\sigma_{\underline{P}}$ has and $D_{\xi}^{\beta} \sigma_{\underline{p}}$ is a polynomial in $\frac{\underline{p}}{\xi}$ of degree $m-|\beta|$. To get general pseudo-differential operators, we impose on $\sigma_{\underline{p}}$ the conditions 


$$
\left|D_{x}^{\alpha} D_{\xi}^{\beta} \sigma_{\underline{p}}(x, \xi)\right| \leq C_{\alpha \beta L}(1+|\xi|)^{m}-|\beta|,
$$

for $x \in L$, $L$ being an arbitrary compact set; but this time $m$ is any real number. We then say that $\sigma_{\underline{p}}$ is a symbol of order $m$ and $\underline{P}$ is a pseudodifferential operator of order $m$. The case when $\sigma_{\underline{p}}=\sigma_{\underline{p}}^{0}+\sigma_{\underline{p}}^{1}$, $\sigma_{\underline{p}}^{1}$ being a symbol of order < $m$ and $\sigma_{\underline{p}}^{\circ}$ is positively homogeneous of degree $m$ in $\xi$ is the most interesting one. We thein say that $\sigma_{\underline{p}}^{o}$ is the principal part of $P$. The essential properties of these operators are as follows:

I) If $\sigma_{\underline{P}}$ is defined for $x \in \Omega, \xi \in \underline{R}^{n}, \underline{P} \cdot u$ is defined for $u \in \underline{D}(\Omega)$, and we have $\underline{P} \cdot u \in \underline{E}(\Omega)$.

II) If $\underline{P} \cdot u \in \underline{D}(\Omega)$ for $u \in \underline{D}(\Omega)$, $\underline{Q P}$ is defined and is a pseudo-differential operator of order $m+r$ for all pseudo-differential operators $Q$ of order $r$; and furthermore if $Q$ admits a principal part, then it is also true for $\mathrm{QP}$ and we have the relation (4).

III) If $m<-n, \underline{P}$ is an integral operator, and if $m<-n-r$ for an integer $r>0$, the kernel of $\underline{p}$ is of class $C^{r}$. As it is well known that integral operators are much more manageable than differential operators, we thus conclude that a pseudo differential operator is all the better when $\mathrm{m}$ is negative and large in absolute value.

7. We would like to invert a pseudo-differential operator $\underline{P}$ of order $m$ by a pseudo-differential operator $Q$ of order $-m$; but the relation $Q P=I$, Implies $\sigma_{\underline{Q}}^{0} \cdot \sigma_{\underline{p}}^{0}=1$ and consequently we must have $\sigma_{\underline{p}}^{0}(x, \xi) \neq 0$ for $\xi \neq 0$; these operators are called elliptic operators and these are the only ones for which we can hope to find an inverse by these methods.

Now comes the idea of a parametrix, which dates back to E.E. Leyi and Hilbert (1907). We are not looking for a true inverse of $\underline{p}$ but only a pseudo-differential operator $Q$ that is an approximate inverse of $\underline{P}$ in the sense that $\underline{P Q}=\underline{I}+\underline{R}$ where $\underline{R}$ is "less singular" than $\underline{P}$. To obtain a 
solution of $\underline{P} \cdot u=f$, we try to find it in the form $u=Q \cdot v$. This yields $\mathrm{v}+\underline{\mathrm{R}} \cdot \mathrm{v}=\mathrm{f}$; if $\underline{\mathrm{R}}$ is an integral operator we then arrive at an integral equation which in principle is more manageable than a partial differential equation.

8. This idea has been realized with the aid of the theory of Schwartz's Kernels. For any pseudo-differential operator $\underline{P}$ we can define $\underline{P} \cdot T$ for any distribution $\mathrm{T}$, with compact support, $\underline{\mathrm{P}} \cdot \mathrm{T}$ evidently being a distribution. Now, among the "good" pseudo-differential operators, there are some "better than others"; these are the integral operators with $\mathrm{C}^{\infty}$ Kernels, called smoothing operators; they are characterized by the fact that $\underline{K} . T \in \underline{E}(\Omega)$ for all distributions $T \in \underline{E}^{\prime}(\Omega)$ (in a nutshell, we may say that $\underline{\mathrm{K}}$ transforms the worst distributions into the best possible ones); for all pseudo-differential operators $\underline{\mathrm{P}}, \underline{\mathrm{PK}}$ and $\underline{\mathrm{KP}}$ are also smoothing operators. The existence theorem of a parametrix can then be made more precise as follows: if $\underline{p}$ is an elliptic pseudo-differential operator of order $\mathrm{m}$, then there is an elliptic pseudodifferential operator $Q$ of order $-m$ satisfying $\underline{Q P}=\underline{I}+\underline{R}$ and $\underline{P Q}=\underline{I}+\underline{R}$ ', where $\underline{R}$ and $\underline{R}^{\prime}$ are smoothing operators. We then not only conclude the possibility of obtaining solutions of $\underline{P} \cdot u=f$ with the help of integral equations, but also the fundamental property of hypoelliplicity of elliptic operators: if $T \in \underline{E^{\prime}}(\Omega)$ is such that $\underline{\mathrm{P}} \cdot \mathrm{T}=\mathrm{f} \in \underline{\mathrm{E}}(\Omega)$, it follows that $T+\underline{R} \cdot T=\underline{Q} \cdot f \in \underline{E}(\Omega)$, and since $\underline{R}$ is smoothing, we have also that $\underline{R} \cdot T \in$ $E(\Omega)$, from which we finally conclude that $T \in \underline{E}(\Omega)$.

9. The idea of the proof of the existence theorem of parametrices is particularly interesting; the fact that smoothing operators form a two-sided ideal in the algebra of pseudo-differential operators suggests calculating modulo this ideal: we then write $\underline{\mathrm{P}}_{1} \sim \underline{\mathrm{P}}_{2}$ if $\underline{\mathrm{P}}_{1}-\underline{\mathrm{P}}_{2}$ is smoothing. The hypothesis that $\sigma_{\underline{p}}^{o}(x, \xi) \neq 0$ for $\xi \neq 0$ leads us to take as the first 
approximation to the parametrix namely a pseudo-differential operator $\underline{Q}^{\circ}$ such that $\sigma_{\underline{Q}_{0}}^{\circ}=\left(\sigma_{\underline{P}}^{0}\right)^{-1}$; we then find $\underline{Q}_{0} \underline{P}=\underline{I}-\underline{P}_{1}$, where $\underline{P}_{1}$ is of order -1 , and it remains to find $\underline{Q}_{1}$ such that $\underline{Q}_{1}\left(\underline{I}-\underline{P}_{1}\right)=\underline{I}+\underline{R}$, where $\underline{R}$ is smoothing. The natural reaction in all problems of this type is to write

$$
\underline{Q}_{1}=\underline{I}+\underline{P}_{1}+\underline{P}_{1}^{2}+\ldots \underline{p}_{1}^{k}+\ldots,
$$

which by itself does not have any meaning. But we can give it a meaning as an asymptotic expansion. In general, suppose we have a sequence $\underline{A}_{1}, \underline{A}_{2}, \ldots$ of pseudo-differential operators of orders $m_{1}, m_{2}, \ldots$ respectively, decreasing to $-\infty$; then there exists a pseudo-differential operator A having the following property: for all k, $\underline{A}-\left(\underline{A}_{1}+\underline{A}_{2}+\ldots+\underline{A}_{k}\right)$ is of order $<m_{k}$ and we write this as $\underline{\mathrm{A}} \sim \underline{\mathrm{A}}_{1}+\underline{\mathrm{A}}_{2}+\ldots+\underline{A}_{\mathrm{k}}+\ldots$ In our case we have $\underline{A}_{k}=\underline{\mathrm{P}}_{1}^{\mathrm{k}}$, and so taking $\underline{Q}_{1} \sim \underline{I}+\underline{P}_{1}+\ldots \underline{P}_{1}^{k}+\ldots$, we then conclude that $\underline{R}=\underline{Q}_{1}\left(\underline{I}-\underline{P}_{1}\right)$ is of order $\mathrm{m}$ for all real $\mathrm{m}$, which implies that $\mathrm{R}$ is smoothing.

10. This calculation is but one example of the manageability of pseudodifferential operators. Similarly, if $\sigma_{\underline{p}}^{o}>0$, we can define (modulo smoothing operators) the roots $\underline{P}^{1 / d}$ of $\underline{P}$, and even the irrational powers of $\underline{P}$. We can also continuously vary the pseudo-differential opertors as functions of parameters without being always restricted to using polynomial symbols in $\xi$. Moreover, the development of this theory received its first impetus from the problem of differential topology which led to the celebrated index formula of Atiyah-Singer. This involves operators defined no longer on the open sets of $\underline{R}^{n}$, but on a differential manifold $X$. With the help of charts this definition does not pose any problems for differential operators which are local operators; for pseudo-differential operators which do not possess this property it demands greater care, but there is no essential difficulty. For a compact manifold $X$, these operators are then linear mappings from $\Gamma(E)$ 
into $\Gamma(F)$, where $E$ and $F$ are vector bundles of base $X, \Gamma E$ and $\Gamma F$ being the spaces of $C^{\infty}$ sections of these bundles over $X$. The existence of a parametrix and the spectral theory show that if $\underline{P}$ is a self-adjoint elliptic pseudodifferential operator mapping $\Gamma(E)$ into itself, $\underline{P}$ (which is an unbounded operator in the Hilbert space of square integrable sections), has a real discrete spectrum with $C^{\infty}$ eigenfunctions, and $(\underline{P}-\zeta \underline{I})^{-1}$ is compact if $\zeta$ is not in the spectrum of $\underline{P}$. This result is the starting point of the Hodge and Atiyah-Singer theories as well as of the generalization by Atiyah-Bott of the Lefschetz fixed point formula.

11. Let us briefly deal with the problem of boundary values in the theory of elliptic pseudo-differential operators as dealt with by Calderon and Hormander; this presentation clearly indicates why the Cauchy problem has no solution in general and why potentials appear in the expression of the solution when the latter does exist.

Suppose $\underline{P}$ is an elliptic differential operator of order $m>1$ on an open set $M \subset \underline{R}^{n}$ with $n \geq 3$; it can be shown that $m$ is necessarily even. Let $\Omega$ be an open bounded set such that $\bar{\Omega} \subset \mathrm{M}$ with smooth boundary $\partial \Omega$ for all functions $u \in E(M)$, we denote by $C D_{m}(u)$ (Cauchy date on $\partial \Omega$ ) the vector function $\left(u, \frac{\partial u}{\partial n}, \frac{\partial^{2} u}{\partial n^{2}}, \ldots . \frac{\partial^{m-1} u}{\partial n^{m-1}}\right)$ on $\partial \Omega$ (normal derivatives). In a distributional sense we can write

$$
\underline{\mathrm{P}} \cdot\left(\mathrm{u} \phi_{\Omega}\right)=(\underline{\mathrm{P}} \cdot \mathrm{u}) \phi_{\Omega}+\underline{\mathrm{N}} \underline{\mathrm{P}} \mathrm{CD}_{\mathrm{m}}(\mathrm{u})
$$

where $g i \underline{N}_{\underline{P}} \cdot g$ is an operator which associates to a function $g \in\left(\underline{E}(\partial(\Omega))^{m}\right.$ a distribution with support in $\partial \Omega$. If $\underline{Q}$ is a parametrix of $\underline{P}$ in $M$, we can show that for all functions $f \underline{D}(M)$, the distribution $Q$ ( $f_{\Omega}$ ) has a restriction to $\Omega$ which extends to a function of $E(\bar{\Omega})$ (Q being the volume potential). Similarly, for all functions $g \in(\underline{E}(\partial \Omega))^{\mathrm{m}}, \underline{Q} \cdot(\underline{\mathrm{N}} \underline{\mathrm{P}} \cdot \mathrm{g})$ extends 
to a function in $\underline{E}(\bar{\Omega})$ (Q being a multi- layer potential). Suppose for simplicity that $\underline{Q P}=\underline{P Q}=\underline{I}$ on $\underline{E}^{\prime}(M)$, and that we have for $u \in \underline{E}(\bar{\Omega})$

$$
\left.\begin{array}{l}
\underline{P} \cdot u=f \in \underline{E}(\bar{\Omega}) \\
C D_{m}(u)=g \in(\underline{E}(\partial \Omega))^{m}
\end{array}\right\}
$$

we can conclude by equation (6) that over $\Omega$ we have

$$
\mathrm{u}=\underline{Q} \cdot\left(\mathrm{f}_{\Omega}\right)+\underline{Q} \cdot\left(\underline{\mathrm{N}}_{\underline{\mathrm{p}}} \cdot(\mathrm{g})\right),
$$

the sum of a volume potential and multi-layer potential; moreover if we suppose that $u$ satisfies the boundary conditions of (7), we arrive at the necessary condition

$$
g-\underline{C} \cdot g=\mathrm{CD}_{\mathrm{m}}\left(\underline{Q} \cdot\left(f \phi_{\Omega}\right)\right),
$$

relating $\mathrm{f}$ and $\mathrm{g}$ where $\underline{\mathrm{C}}: \mathrm{g} \rightarrow \mathrm{CD}_{\mathrm{m}}(\underline{\mathrm{Q}} \cdot(\underline{\mathrm{N}} \underline{\mathrm{P}} \cdot \mathrm{g}))$ is called the Calderon operator on $\partial \Omega$ corresponding to $\underline{P}$ and $Q$. A deeper study of this operator shows that (9) imposes $\frac{m}{2}$ linear conditions. The classical Cauchy problem for $\Omega$ has therefore no solution in general, and to obtain a reasonable problem, instead of $m$ boundary conditions in (7) we must impose only $\frac{m}{2}$ linear conditions :

$$
\underline{B}_{j} \cdot C D_{m}(u)=g_{j}\left(1 \leq j \leq \frac{m}{2}\right),
$$

where $\underline{B}_{j}$ are pseudo-differentlal operators on $\partial \Omega$ (if we take $\underline{B}_{j} \cdot C D_{m} u$ $=\frac{\partial^{j-1} u_{j}}{\partial n^{j-1}}$, we obtain the Dirichlet problem as formulated by Gärding-Višsik).

The study of the boundary-value problems of type (10) for $\underline{P}$ introduces ellipticity conditions on the $B_{j}$ (Shapiro-Lopatinski) which imply that the mapping

$$
\underline{A}: \quad u \rightarrow\left(\underline{P} \cdot u,\left(\underline{B}_{j} \cdot C D_{m} u\right)\right)\left(1 \leq j \leq \frac{m}{2}\right) \text { from } \underline{E}(\bar{\Omega}) \text { into } \underline{E}(\bar{\Omega}) \times\left(\underline{E}(\partial \Omega)^{m / 2}\right.
$$


has a kernel of finite rank and has closed range of finite co-dimension.

But the analogy with the Fredholm theory stops here: it is in fact possible that the problem

$\underline{\mathrm{P}} \cdot \mathrm{u}=\lambda \mathrm{u}, \underline{\mathrm{B}} \mathrm{g} \cdot \mathrm{CD}_{\mathrm{m}} \mathrm{u}=0\left(1 \leq \mathrm{j} \leq \frac{\mathrm{m}}{2}\right)$ has non-zero solutions for all $\lambda \in \underline{\mathrm{C}}$, in other words, we have a "spectrum" equal to the-whole plane $\underline{C}$ instead of a discrete spectrum. To get a discrete spectrum we must impose on $\underline{\mathrm{P}}$ Hermitian conditions.

12. The idea that leads to the pseudo-differential operators is open to extensive generalizations, which enable us to tackle non-elliptic problems. The definition (2) would lead us to write, taking into account the definition of Fourier transforms

$$
\begin{aligned}
& \int_{\underline{R}^{n}} e^{2 \pi i(x \mid \xi)} \sigma(x, \xi) F u(\xi) d \xi \\
& =\int_{\underline{R}^{n}} \int_{x \underline{R}^{n}} e^{2 \pi i(x-y \mid \xi)} \sigma(x, \xi) u(y) d y d \xi,
\end{aligned}
$$

but in general the double integral does not have any meaning even if $u \in \underline{D}\left(\underline{R}^{n}\right)$. However, we can define it as an improper integral, a notion that the theory of distributions allows us to reinstate. More general1y we can define in the same way the "oscillating integrals"

$$
\iint e^{i \Phi(x, y, \xi)} a(x, y, \xi) d y d \xi,
$$

where the derivatives of $\underline{a}$ are limited by inequalities of type (5), and $\Phi$ is a "phase function" subject to conditions which amount to saying that except for certain singular points $(x, y)$, the "angle" $\Phi$ rotates rapidly enough when $|\xi|$ tends to $+\infty$. 
As a classical example, we have the formula which solves the Cauchy problem for the wave equation

$$
\iint e^{2 \pi i((x-y \mid \xi) \pm|\xi| t)\left(u_{0}(y)+\frac{u_{1}(y)}{|\xi|}\right) d y d \xi .}
$$

The introduction of such operators has brought significant progress in the past few years, notably concerning hyperbolic equations and the local existence problem (Lax-Maslov, Hormander-Duistermaat, Treves-Nirenberg, Beals-Fefferman, etc.). 


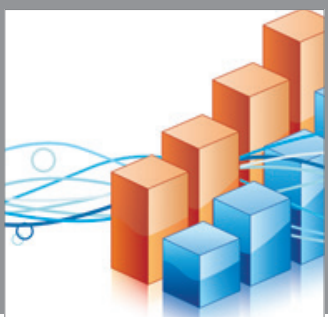

Advances in

Operations Research

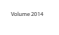

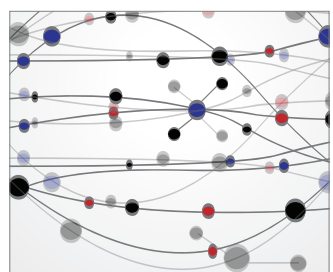

\section{The Scientific} World Journal
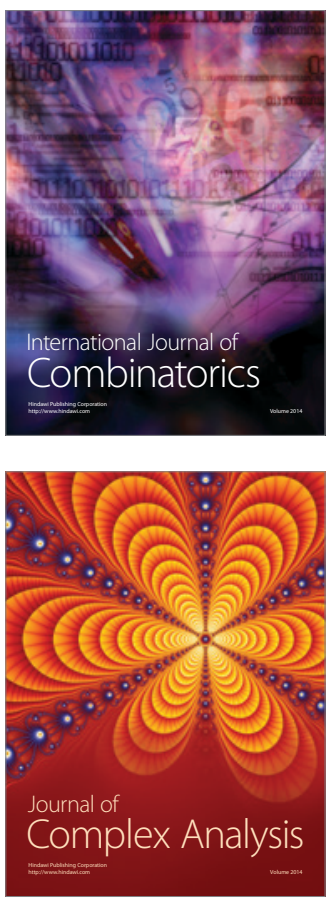

International Journal of

Mathematics and

Mathematical

Sciences
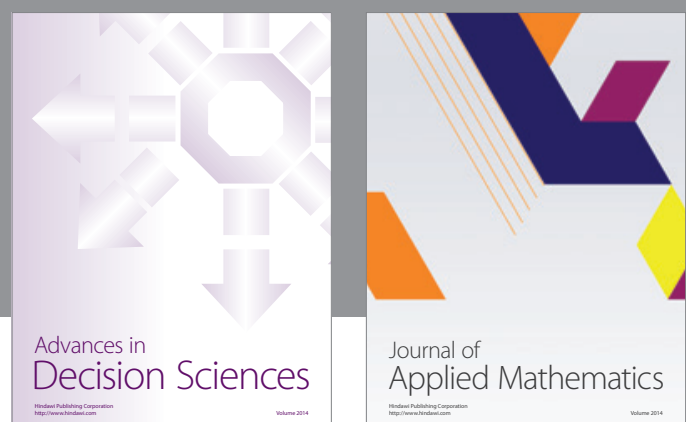

Journal of

Applied Mathematics
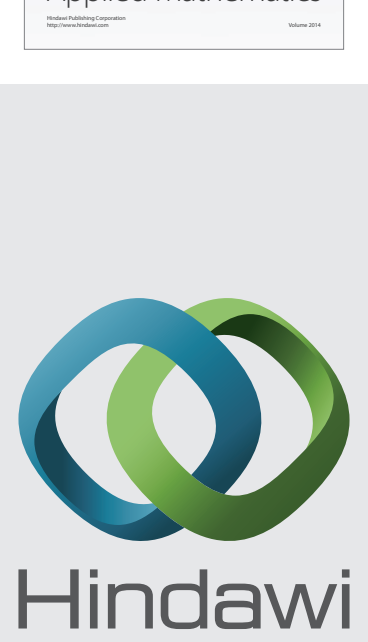

Submit your manuscripts at http://www.hindawi.com
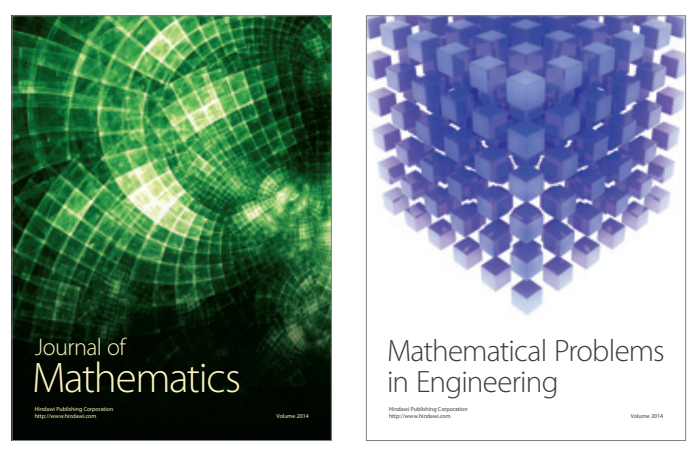

Mathematical Problems in Engineering
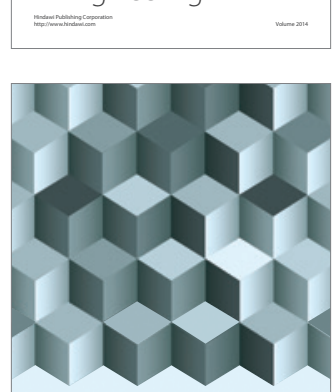

Journal of

Function Spaces
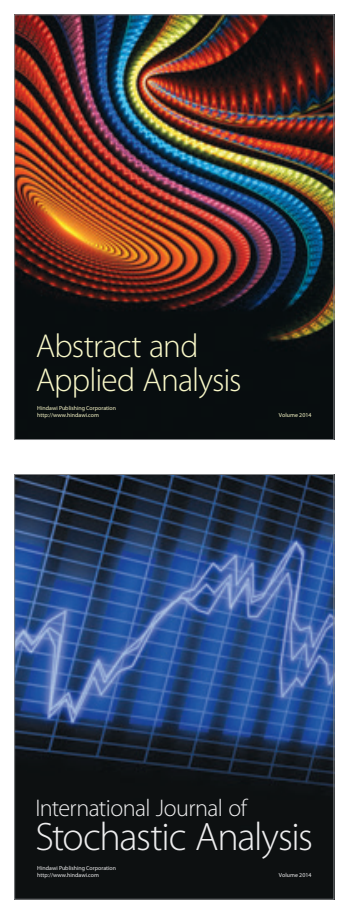

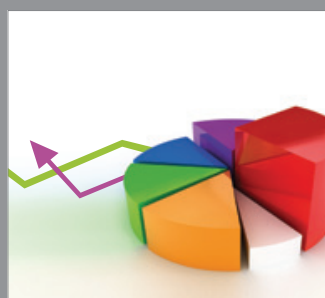

ournal of

Probability and Statistics

Promensencen
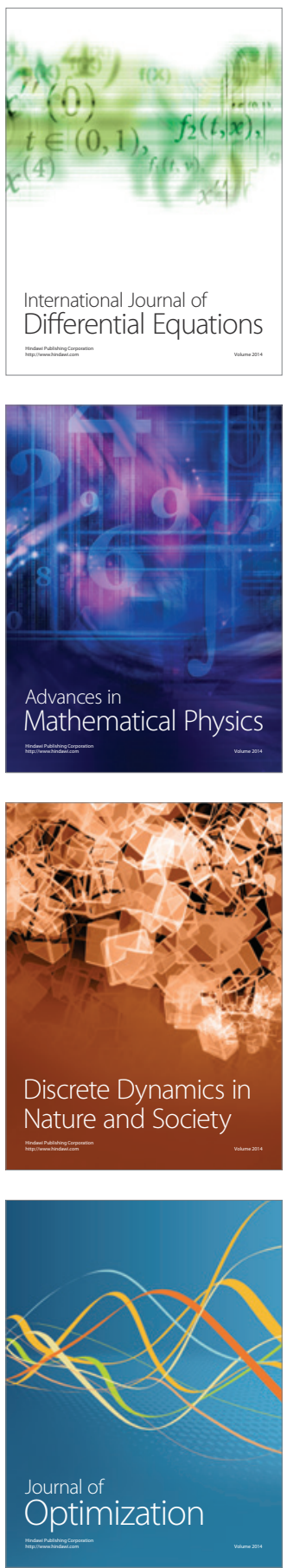